\title{
Chemical and Mechanical Properties of Cuticular Membranes Isolated from Young Matured Leaves of Sonneratia alba
}

\author{
Yuki Takahashi, Shuntaro Tsubaki, Masahiro Sakamoto, Shin Watanabe, Wahyu Dwianto, \\ and Jun-ichi Azuma
}

\begin{abstract}
Leaf cuticular membranes (CMs) of Sonneratia alba are considered to play an important role in environmental tolerance, and chemical and mechanical properties of their CMs are crucial factors which provide protective barrier and mechanical supports. Leaf CMs were enzymatically isolated from both adaxial and abaxial sides of young matured leaves $\left(\mathrm{L}_{3}\right)$, and their chemical and mechanical properties were compared. Chemical compositions of both CMs were similar reflecting their morphological similarity. The adaxial CM was consisted of $23.4 \%$ wax, $64.2 \%$ cutin, $7.4 \%$ cutan and $6.1 \%$ polysaccharides with thickness of $9 \mu \mathrm{m}$, and the abaxial CM had the values of $23.9 \%, 63.2 \%, 9.4 \%, 4.2 \%$ and $8 \mu \mathrm{m}$, respectively. Major monomers in both cutins were 9,10-epoxy-18-hydroxyoctadecanoic acid and 9(10),16-dihydroxyhexadecanoic acid. Mechanical properties of both CMs were also similar reflecting their morphological and chemical compositional similarities.
\end{abstract}

Key words: mangrove, Sonneratia alba, young matured leaves, cuticular membrane, cutin, biomechanics.

\section{Introduction}

Mangrove trees grow intertidal zones of tropical and subtropical areas showing zonal distributions parallel to the coastline. Sonneratia alba is a kind of mangrove species which widely distributed from East Africa to Southeast and East Asia and tropical Australia. They are generally distributed outermost area of intertidal zones with high environmental tolerance and often form a seaward fringe (Tomlinson 1986).

Aerial parts of higher plants are covered with a protective barrier, cuticle or cuticular membrane (CM), which consists of soluble wax fraction, aliphatic biopolyester cutin, persistent polymer called cutan and polysaccharides (Jeffree 1996; Heredia 2003). Plant CMs minimize solute diffusion and uncontrolled water loss, prevent pathogen penetration and also contribute to mechanical support of plant body (Bargel et al. 2006; Heredia 2003). Therefore, chemical and mechanical properties of $\mathrm{CMs}$ are determinants of their functions. In dorsiventral dicotyledonous plants, adaxial and abaxial sides of leaves have clearly different structure and chemical and mechanical properties (Azuma et al. 2010). In contrast, S. alba is known to have isolateral leaves (Tomlinson 1986). This may give a merit to live in the frontier of intertidal zone under strong sunlight, wind and tide. In this study, CMs were isolated from both adaxial and abaxial sides of leaves of $S$. alba and their chemical and mechanical properties were compared to give a proof of high environmental tolerance of $S$. alba.

\section{Materials and Methods}

Plant Material and Isolation of the CMs

Leaves of $S$. alba were obtained at Iriomote Island,
Okinawa, Japan in May 2009 and February 2010. Leaves after budburst were consecutively numbered from the top in the same shoot. Matured and not aged leaves of $L_{3}$ were used in this study. Leaf CMs were enzymatically isolated with fungal pectinase $(2 \%$, w/v, Aspergillus niger, SigmaAldrich, St. Louis, MO, USA) and Meicelase $(0.5 \%$, w/v, Trichoderma viride, Meiji Seika Kaisha Ltd., Tokyo, Japan). After incubation for $72 \mathrm{~h}$ at $36^{\circ} \mathrm{C}$ in sodium acetate buffer (5 $\mathrm{mM}, \mathrm{pH}$ 5.0) with addition of $6 \%$ ethanol to prevent microbial growth, CMs were peeled off, washed with distilled water, and air-dried.

\section{Morphological and Spectroscopic Analysis}

Isolated CMs were morphologically characterized with low-voltage scanning electron microscopy (LV-SEM, VE8800 , Keyence Co., Osaka, Japan), at 500-fold magnification with $1.7 \mathrm{kV}$ of accelerating voltage. Chemical properties of CMs were characterized with FT/IR (FT/IR4100, JASCO Co., Tokyo, Japan) in $2.0 \mathrm{~cm}^{-1}$ of resolution, and analytical range of $400 \sim 4000 \mathrm{~cm}^{-1}$ by the thin-film method. The $\mathrm{CMs}$ were also analyzed with solid-state CP/MAS ${ }^{13} \mathrm{C}$ NMR (Chemagnetics CMX-300 spectrometer, JEOL Ltd., Tokyo, Japan) operating at $74.7 \mathrm{MHz}$. The spinning rate was $4.5 \mathrm{kHz}$, contact time $2 \mathrm{~ms}$, acquisition time $34 \mathrm{~ms}$, sweep width $30 \mathrm{kHz}$ and pulse delay time $5 \mathrm{~s}$.

\section{Chemical Analyses}

Chemical compositions of the CMs were analyzed by solvent extraction and gravimetric analyses. First, cuticular wax was removed by refluxing the isolated $\mathrm{CMs}$ in a mixture of chloroform and methanol $(2: 1, \mathrm{v} / \mathrm{v})$ for $2 \mathrm{~h}$ at $50^{\circ} \mathrm{C}$. Then, cutin was saponified by refluxing dewaxed $\mathrm{CMs}$ with $1 \%$ $\mathrm{KOH}$ in methanol for $2 \mathrm{~h}$ at $70^{\circ} \mathrm{C}$. Decutinized residues were thoroughly washed with methanol and hydrolyzed by Saeman hydrolysis (Saeman et al. 1945). Constitutive 
monosaccharides were obtained in the filtrate, and the final non-saponifiable and non-hydrolysable residue was defined as cutan. Polysaccharide contents were quantified by the phenol-sulfuric acid method.

Analysis of cutin monomers was conducted according to the method of Walton and Kolattukudy (1972). Reduction of polyester components was carried out by refluxing the dewaxed $\mathrm{CMs}$ in tetrahydrofuran with an excess amount (2.5 times, w/w) of $\mathrm{LiAlH}_{4}$ for $48 \mathrm{~h}$ at $70^{\circ} \mathrm{C}$. Reduction was also carried out with $\mathrm{LiAlD}_{4}$ to label carboxyl and epoxy groups with deuterium. Reduced monomers were extracted with diethyl ether, dehydrated with anhydrous sodium sulfate, and evaporated to dryness under reduced pressure. After TMS derivatization with N,O-bis(trimethylsilyl)acetamide, the compositions of the derivatives were determined by GC-MS (GC/MS 2010/PURVUM 2, Shimazu Co., Kyoto, Japan) with a DB-1 $(0.25 \mathrm{~mm} \times 30 \mathrm{~m}$, df $=0.25$ $\mu \mathrm{m})$ non-polar capillary column ( $\mathrm{J}$ W Scientific, Agilent Technologies, Inc., Santa Clara, CA, USA) and a helium carrier gas at a flow rate of $0.91 \mathrm{~mL} / \mathrm{min}$. The column oven temperature was programmed from $195^{\circ} \mathrm{C}$ to $240^{\circ} \mathrm{C}$ at the rate of $2^{\circ} \mathrm{C} / \mathrm{min}$, and then held for $10 \mathrm{~min}$, and subsequently heated up to $300^{\circ} \mathrm{C}$ at the rate of $10^{\circ} \mathrm{C} / \mathrm{min}$. The interface temperature was $250^{\circ} \mathrm{C}$, and electron impact (EI) ionization was conducted at $70 \mathrm{eV}$. The mass spectra were obtained in the range of $40.00 \sim 650.00 \mathrm{~m} / \mathrm{z}$ by scanning mode.

\section{Mechanical Tests}

Mechanical properties of the isolated CMs were measured by the tensile test with a tensile tester (Tack Tester TA-500, UBM Co., Kyoto, Japan). Rectangular uniform segments $(5 \times 20 \mathrm{~mm})$ of samples were clipped with stainless cramps, and deformed at a constant rate at room temperature $\left(23^{\circ} \mathrm{C}\right)$. The data of strain and load were collected twice a second. Elastic modulus $E(\mathrm{MPa})$ was obtained from linear region at the initial part of the stressstrain curve. Breaking stress $\sigma_{\max }(\mathrm{MPa})$ and maximum strain $\varepsilon_{\max }(\%)$ were also determined for each sample.

\section{Statistics}

Results were presented as mean \pm standard deviation (SD). Contents of wax and cutin were calculated as the average of $8 \sim 10$ replicates, and 3 6 replicates were used to obtain the contents of cutan and polysaccharide. Composition of reduced cutin monomers was determined as the average of triplicate analyses. Biomechanical parameters were calculated as the average of $6 \sim 10$ specimens prepared from 5 10 replication sets. Mean values of adaxial and abaxial CMs were compared by $t$-tests at the $5 \%$ level of significance.

\section{Results and Discussion}

\section{Morphological and Spectroscopic Properties}

Young matured leaves $\left(L_{3}\right)$ of $S$. alba had stomatal wrinkled surface in both adaxial and abaxial sides as shown in Figure 1. Thickness of the adaxial CMs was $9 \pm 2 \mu \mathrm{m}$ and their yield was $0.76 \pm 0.24 \%$ of the leaves in the dry state, and the values for the abaxial CMs were $8 \pm 2 \mu \mathrm{m}$ and $0.69 \pm 0.24 \%(n=20)$. CMs of $S$. alba leaves were well-developed throughout their surfaces. Their contents were, however, low due to succulence. As shown in Figure 2 (A), FT-IR spectra of the isolated CMs had predominant absorptions at 2920, 2850 and $1730 \mathrm{~cm}^{-1}$ assigned to the asymmetric and symmetric stretching vibrations of the methylene groups and the stretching vibrations of ester carbonyl groups (Ramirez et al. 1992; Villena et al. 2000), confirming aliphatic polyester-nature of the CMs. In addition, the CP/MAS ${ }^{13} \mathrm{C}$ NMR spectra showed presence of carbonyl (160 180 ppm), carbohydrate (60 110 ppm), $\mathrm{OCH}_{3}$ (around $56 \mathrm{ppm}$ ) and alkyl carbons (10 45 ppm) as shown in Figure 2 (B) (Pacchiano et al. 1993; Tsubaki et al. 2008). These results clearly show that both adaxial and abaxial CMs are structurally similar.

\section{Chemical Compositions}

Chemical compositions of the isolated CMs were listed in Table 1. Contents of wax, cutin, cutan and polysaccharides accounted for $23.4,64.2,7.4$ and $6.1 \%$, respectively, in the adaxial $\mathrm{CM}$. In the abaxial $\mathrm{CM}$, the values accounted for $23.9,63.2,9.4$ and $4.2 \%$, respectively. The CMs from leaves of $S$. alba were abundant in cutin and wax. No significant differences were detected in the values between adaxial and abaxial CMs, supporting results of spectroscopic analyses.

Since cutin was the most abundant constituent of the $\mathrm{CM}$, an attention was focused on the monomer composition of the cutin. Monomeric compositions of both cutins identified according to Walton and Kolattukudy (1972) were summarized in Table 2. Octadecan-3-ol was the predominant component followed by hexadecan-3-ol. By combination with GC/MS analysis of the deuterium-labeled monomers, these components were concluded to be derived from 9,10-epoxy-18-hydroxyoctadecanoic acid and 16-dihydroxyhexadecanoic acid (obtained as a mixture of 9and 10-isomers), respectively. They are detected as common monomers of cutins in the other dicotyledonous plants (Walton and Kolattukudy 1972; Kolattukudy 1980). Both adaxial and abaxial cutins were compositionally similar. As a summary, present results showed isolaterality of leaves of $S$. alba for the first time not only from morphological point of view but also from chemical compositional properties. 


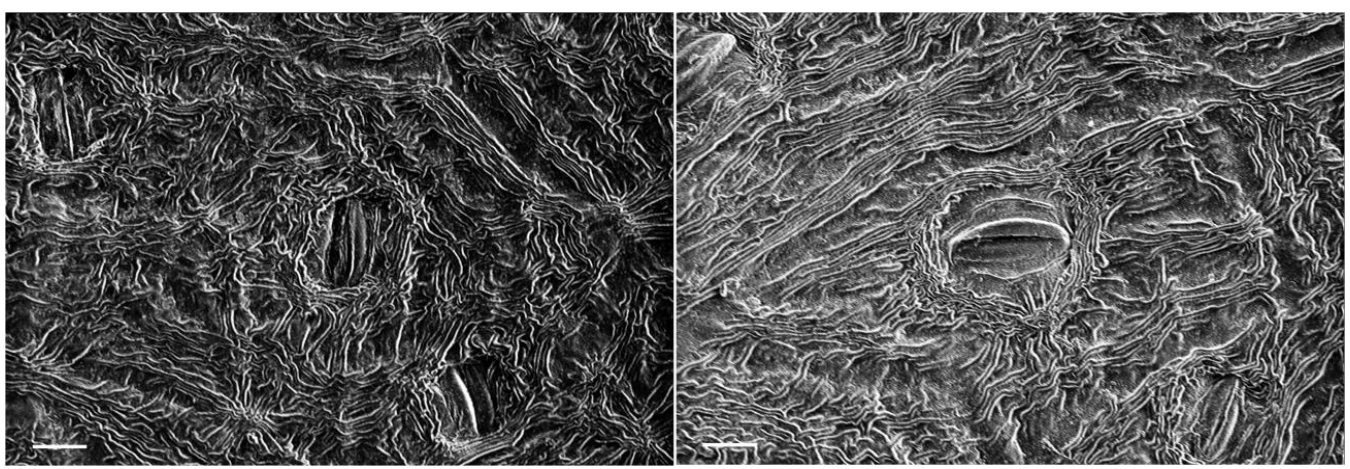

Figure 1. Typical LV-SEM images of the CMs isolated from a young matured leaf $\left(\mathrm{L}_{3}\right)$ of $S$. alba (left, adaxial side; right, abaxial side; $\times 500$; bar equals $20 \mu \mathrm{m}$ ).

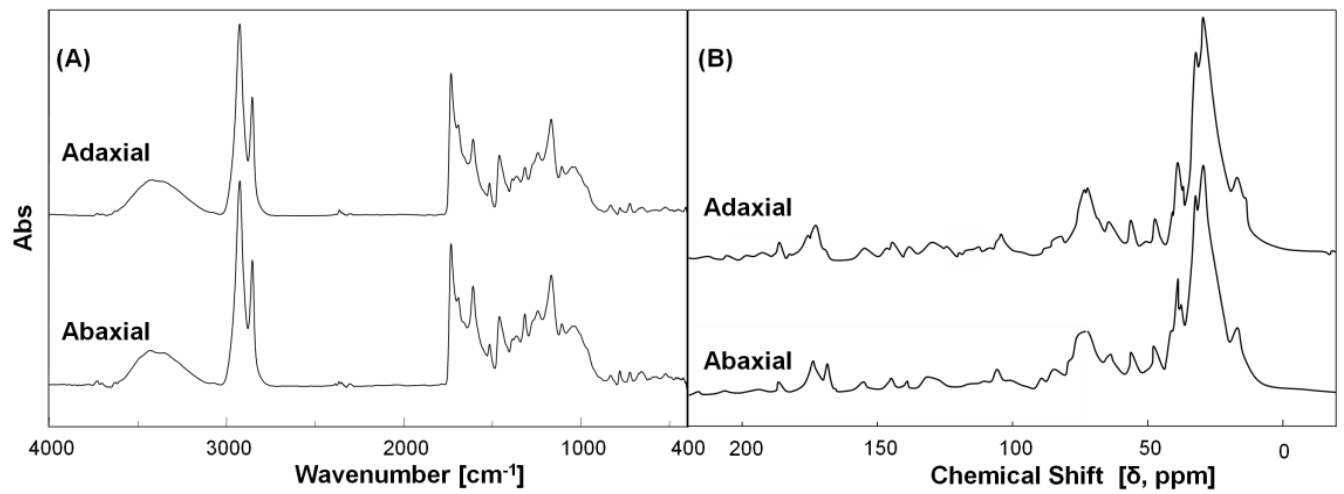

Figure 2. Typical (A) FT-IR spectra and (B) solid-state CP/MAS ${ }^{13} \mathrm{C}$ NMR spectra of the CMs isolated from young matured leaves $\left(L_{3}\right)$ of $S$. alba.

Table 1. Chemical composition of the CMs isolated from young matured leaves $\left(L_{3}\right)$ of $S$. alba. (w\%, Mean $\left.\pm S D\right)$.

\begin{tabular}{lrr}
\hline & \multicolumn{1}{c}{ Adaxial } & Abaxial \\
\hline Wax $^{*}$ & $23.4 \pm 4.1$ & $23.9 \pm 4.1$ \\
Cutin $^{*}$ & $64.2 \pm 2.6$ & $63.2 \pm 4.5$ \\
Cutan $^{* *}$ & $7.4 \pm 3.1$ & $9.4 \pm 3.2$ \\
Polysaccharide $^{* *}$ & $6.1 \pm 1.3$ & $4.2 \pm 2.3$ \\
\hline
\end{tabular}

${ }^{*} n=8 \sim 10,{ }^{*} n=3 \sim 6$

Table 2. Composition of the reduced monomers obtained by hydrogenolysis of cutin. ( $R$, retention time in min; Relative area $\%$, Mean $\pm \operatorname{SD}(n=3))$.

\begin{tabular}{cclrr}
\hline No. & $R_{t}(\mathrm{~min})$ & \multicolumn{1}{c}{ Components } & \multicolumn{1}{c}{ Adaxial } & \multicolumn{1}{c}{ Abaxial } \\
\hline 1 & 6.5 & Hexadecan-1-ol $\left(\mathrm{C}_{16: 0}\right)$ & $0.18 \pm 0.11$ & $0.31 \pm 0.09$ \\
2 & 10.2 & Octadecan-1-ol $\left(\mathrm{C}_{18: 0}\right)$ & $0.03 \pm 0.03$ & $0.11 \pm 0.04$ \\
3 & 14.1 & Hexadecan-2-ol $\left(\mathrm{C}_{16: 0}\right)$ & $0.77 \pm 0.17$ & $0.84 \pm 0.06$ \\
4 & 18.7 & Octadecen-2-ol $\left(\mathrm{C}_{18: 1}\right)$ & $1.85 \pm 0.20$ & $1.73 \pm 0.15$ \\
5 & 19.4 & Hexadecan-3-ol $\left(\mathrm{C}_{16: 0}\right)$ & $25.57 \pm 1.14$ & $18.14 \pm 0.64$ \\
6 & 20.7 & Tetradecen-3-ol $\left(\mathrm{C}_{14: 1)}\right.$ & $2.91 \pm 0.18$ & $3.31 \pm 0.18$ \\
7 & 22.3 & Heptadecan-3-ol $\left(\mathrm{C}_{17: 0}\right)$ & $0.27 \pm 0.03$ & $0.23 \pm 0.01$ \\
8 & 24.9 & Octadecen-3-ol $\left(\mathrm{C}_{18: 1}\right)$ & $0.79 \pm 0.05$ & $1.60 \pm 0.15$ \\
9 & 25.7 & Octadecan-3-ol $\left(\mathrm{C}_{18: 0}\right)$ & $47.48 \pm 1.21$ & $47.87 \pm 0.59$ \\
10 & 27.4 & Hexadecen-3-ol $\left(\mathrm{C}_{16: 1)}\right.$ & $3.29 \pm 0.68$ & $7.84 \pm 0.24$ \\
11 & 31.1 & Octadecan-4-ol $\left(\mathrm{C}_{18: 0}\right)$ & $2.75 \pm 0.01$ & $4.12 \pm 0.05$ \\
& & Unidentified & $14.11 \pm 0.31$ & $10.12 \pm 0.43$ \\
\hline
\end{tabular}




\section{Mechanical Properties}

Biomechanical parameters of the isolated $\mathrm{CMs}$ were analyzed and the results of breaking stress $\left(\sigma_{\max }\right)$, maximum strain $\left(\varepsilon_{\max }\right)$ and elastic modulus $(E)$ were listed in Table 3. Although the values of $\sigma_{\max }, \varepsilon_{\max }$ and $E$ of abaxial CMs were slightly higher than those of adaxial CMs, no significant differences were statistically observed due to large variances. Both adaxial and abaxial CMs thus showed similar mechanical properties, as expected from their similarities in morphological and chemical properties.

Table 3. Mechanical properties of the CMs isolated from young matured leaves $\left(\mathrm{L}_{3}\right)$ of $S$. alba (Mean $\pm \mathrm{SD}$ $(n=6 \sim 10))$.

\begin{tabular}{lrr}
\hline & \multicolumn{1}{c}{ Adaxial } & \multicolumn{1}{c}{ Abaxial } \\
\hline Breaking Stress $\sigma_{\max }(\mathrm{MPa})$ & $6.47 \pm 1.99$ & $7.34 \pm 1.37$ \\
Maximum Strain $\varepsilon_{\max }(\%)$ & $4.34 \pm 1.96$ & $4.50 \pm 1.55$ \\
Elastic Modulus $E(\mathrm{MPa})$ & $303 \pm 43$ & $364 \pm 48$ \\
\hline
\end{tabular}

\section{Conclusions}

Both adaxial and abaxial CMs enzymatically isolated from the leaves of $S$. alba $\left(\mathrm{L}_{3}\right)$ were found to have similar spectroscopic and chemical compositional and mechanical properties. These properties may participate to the high environmental tolerance of $S$. alba enough to survive at the frontier of intertidal zones.

\section{References}

Azuma, J.; S. Tsubaki; R. Yudianti; M. Karina. 2010. Characterization of Cuticle Layer of Ilex latifolia. Wood Research Journal 1: 56-63.

Bargel, H.; K. Koch; Z. Cerman; C. Neinhuis. 2006 Structure-function Relationships of the Plant Cuticle and Cuticular Waxes - A Smart Material? Functional Plant Biology 33: 893-910.

Heredia, A. 2003. Biophysical and Biochemical Characteristics of Cutin, A Plant Barrier Biopolymer. BBA-Gen. Subjects 1620: 1-7.

Jeffree, C.E. 1996. Structure and Ontogeny of Plant Cuticles. In: Plant Cuticles: An Integrated Functional Approach. Kerstiens, G., Ed. BIOS Scientific Publishers, Oxford: 33-82.

Kolattukudy, P.E. 1980. Biopolyester Membranes of Plants: Cutin and Suberin. Science 208: 990-1000.

Pacchiano, R.A.; W, Sohn; V.L. Chlanda; J.R. Garbow; R.E. Stark. 1993. Isolation and Spectral Characterization of
Plant-Cuticle Polyesters. J. Agric. Food Chem. 41: 7883.

Ramirez F.J.; P. Luque; A. Heredia; M.J. Bukovac. 1992. Fourier-transform IR Study of Enzymatically Isolated Tomato Fruit Cuticular Membrane. Biopolymers 32: 1425-1429.

Saeman J.F.; J.L. Bubl; E.E. Harris. 1945. Quantitative Saccharification of Wood and Cellulose. Ind. Eng. Chem. Anal. Ed. 17: 35-37.

Tomlinson, P.B. 1986. The Botany of Mangroves. Cambridge University Press, Cambridge: 367-371.

Tsubaki S.; H. lida; M. Sakamoto; J. Azuma. 2008. Microwave Heating of Tea Residue Yields Polysaccharides, Polyphenols, and Plant Biopolyester. J. Agric. Food Chem. 56: 11293-11299.

Villena, J.F.; E. Domínguez; A. Heredia. 2000. Monitoring Biopolymers Present in Plant Cuticles by FT-IR Spectroscopy. J. Plant Physiol. 156: 419-422.

Walton, T.J.; P.E. Kolattukudy. 1972. Determination of the Structure of Cutin Monomers by A Novel Depolymerization Procedure and Combined Gas Chromatography and Mass Spectrometry. Biochemistry 11: 1885-1897.

Yuki Takahashi, Shuntaro Tsubaki, Masahiro Sakamoto, and Jun-ichi Azuma*

Division of Environmental Science and Technology,

Graduate School of Agriculture, Kyoto University

Kitashirakawa Oiwake-cho, Sakyo-ku, Kyoto 606-8502, Japan.

${ }^{*}$ Corresponding Author

Tel/Fax : 81-75-753-6465/6471

E-mail : azumajikkais.kyoto-u.ac.jp

Shin Watanabe

Iriomote Station, Tropical Biosphere Research Center, University of Ryukyus

870, Uehara, Taketomi-cho, Yaeyama-gun,

Okinawa 907-1541, Japan.

Tel/Fax : 81-980-85-6560/6830

E-mail : nabeshin@lab.u-ryukyu.ac.jp

Wahyu Dwianto

Research and Development Unit for Biomaterials, Indonesian Institute of Sciences, Bogor 16911, Indonesia

$\mathrm{Tel} / \mathrm{Fax} \quad: 62-21-87914509 / 87914510$

E-mail : wahyudwianto@yahoo.com 